\title{
A single-center analysis of outcomes, risk factors, and new valves in Asian patients treated with early transcatheter aortic valve implantation
}

\author{
Ying Liang', Wei Wang ${ }^{2}$, Xu Wang ${ }^{2}$, Feilong Hei ${ }^{1}$, Yulong Guan ${ }^{1}$ \\ ${ }^{1}$ Department of Extracorporeal Circulation, Fuwai Hospital, Chinese Academy of Medical Sciences \& Peking Union Medical College, Beijing, \\ China; ${ }^{2}$ Department of Structural Heart Disease, Fuwai Hospital, Chinese Academy of Medical Sciences \& Peking Union Medical College, Beijing, \\ China \\ Contributions: (I) Conception and design: Y Liang, W Wang, Y Guan; (II) Administrative support: X Wang, W Wang, F Hei; (III) Provision of study \\ materials or patients: X Wang, W Wang, F Hei; (IV) Collection and assembly of data: Y Liang; (V) Data analysis and interpretation: Y Liang, Y \\ Guan; (VI) Manuscript writing: All authors; (VII) Final approval of manuscript: All authors. \\ Correspondence to: Yulong Guan. Department of Extracorporeal Circulation, Fuwai Hospital, Chinese Academy of Medical Sciences \& Peking Union \\ Medical College, 167 Beilishi Road, Beijing 100037, China. Email: guanyulong@yahoo.com.
}

Background: Transcatheter aortic valve implantation (TAVI) continues to expand as an optimal treatment in Western countries; however, Asian countries have been slower to adopt this procedure. This research aimed to explore the outcomes and status of early TAVI performed at a single center in Asia, and provide comparative outcomes of the newly designed Chinese valves.

Methods: We enrolled 175 consecutive patients who successfully underwent TAVI from September 2012 to January 2018 at Fuwai Hospital (Beijing, China). After a preliminary assessment of age, we included 109 older patients ( $\geq 69$ years) who underwent surgical aortic valve replacement (SAVR) during the same period. The primary endpoint was all-cause mortality. The inverse probability of treatment weighting (IPTW) was used to reduce potential bias. Cox regression was used to identify the risk factors of a poor prognosis.

Results: The TAVI cohort had higher rates of all-cause mortality [11.4\% vs. $2.4 \%$, hazard ratio (HR): 4.79, 95\% confidence interval (CI): 1.47 to 15.57 , IPTW-adjusted $\mathrm{P}=0.009$ ] and permanent pacemaker implantation (PPI; 14.6\% vs. 1.6\%, HR: 9.98, 95\% CI: 2.71 to 36.67 , IPTW-adjusted $\mathrm{P}<0.001$ ) at 3 years than the SAVR cohort. In the multivariable Cox regression analysis based on the entire sample, liver disease was associated with all-cause mortality (HR: 5.080, 95\% CI: 1.067 to $24.174, \mathrm{P}=0.041$ ). A smoking history was associated with an increased risk of postoperative heart failure (HF) (HR: 4.902, 95\% CI: 1.265 to 18.999, $\mathrm{P}=0.022$ ). Additionally, age (HR: $1.141,95 \% \mathrm{CI}: 1.010$ to $1.288, \mathrm{P}=0.034)$ and diabetes (HR: 7.301, 95\% CI: 2.414 to $22.079, \mathrm{P}<0.001)$ were identified as predictors of postoperative stroke. In the new valve subgroups, the 1-year composite endpoints were 38.2\% (Venus A), 35.3\% (TaurusOne), 34\% (J-Valve), and 28\% (VitaFlow) (P=0.857).

Conclusions: Not all TAVI procedures had satisfactory outcomes compared with SAVR when initiated. At first, our center faced some challenges in delivering TAVI, and this is probably one of the reasons why the use of TAVI has developed slowly in Asia. Further investigations are needed to explore the underlying factors precluding the rapid expansion of TAVI in Asia.

Keywords: Transcatheter aortic valve implantation (TAVI); inverse probability of treatment weighting (IPTW); heart failure (HF); stroke; paravalvular leakage

Submitted Nov 14, 2020. Accepted for publication Jun 30, 2021.

doi: $10.21037 / \mathrm{cdt}-20-928$

View this article at: https://dx.doi.org/10.21037/cdt-20-928 


\section{Introduction}

Extraordinary progress has been achieved in the procedure volume and outcomes of transcatheter aortic valve implantation (TAVI), as well as the expansion of indications for this procedure. These advancements are largely due to the great reformation of valves and delivery systems. On 16 August, 2019, in response to the publication of 2 trials with remarkable outcomes $(1,2)$, the United States Food and Drug Administration (FDA) expanded the indications for the selfexpanding Evolut valve and balloon-expandable Sapien 3 valve to include patients in all risk categories. Alternatively, these devices can also be placed in patients at low surgical risk (3). Although the use of transcatheter therapy has been continuously expanding in Western countries, the uptake of this treatment in Asia has been slow (4), and TAVI only reached the shores of Asia in February 2009, in Singapore (5). Early TAVI centers across Asia have found it challenging to gain more experience due to the low case volume (6). Differences in the anatomy of the Asian population, such as smaller aortic and peripheral vessel sizes and a high prevalence of bicuspid aortic valves, might have raised concerns about the feasibility, risk of procedural complications, prosthesis durability, and long-term outcomes following transcatheter treatment of valve heart diseases (7). Between March 2010 and September 2014, a registry of 11 centers in Singapore, Hong Kong, Taiwan, Japan, and Korea reported 848 TAVI cases (8). Some studies have reviewed the multicenter outcomes in these countries to date (9-12), although few studies have reported the status of early TAVI in a single center in Asia. In recent years, several Chinese-designed TAVI devices have emerged, including Venus A (Venus MedTech, Hangzhou, China, approved April 2017) (13), J-Valve (Suzhou Jiecheng Medical Technology, Suzhou, China, approved June 2017) (14), TaurusOne (Peijia Medical, Suzhou, China) (15), and VitaFlow (MicroPort CardioFlow Medtech, Shanghai, China, approved July 2019) (16). Several studies have summarized the characteristics and examined the suitability of these devices for use in the real-world setting $(7,17-19)$. However, the comparative performance of these valves has not yet been investigated in the literature.

Therefore, this retrospective study was designed to explore the outcomes and status of early TAVI in a large cardiovascular center in China, by comparing them with those of traditional surgical aortic valve replacement (SAVR). We also attempted to identify the key risk factors associated with poor outcomes after TAVI, and compared the different types of Chinese-made valves that were applied in this cohort of TAVI patients.

We present the study in accordance with the STROBE reporting checklist (available at https://dx.doi.org/10.21037/ cdt-20-928).

\section{Methods}

\section{Patient selection}

Consecutive patients who were scheduled for elective TAVI between September 2012 and January 2018 in Fuwai Hospital (Beijing, China) were considered for inclusion in this study. After preliminarily estimating the age distribution of patients with TAVI, we found that at least $90 \%$ of patients were over 69 years old. Accordingly, we enrolled elderly patients ( $\geq 69$ years) who underwent SAVR in addition to the TAVI cohort.

Patients who withdrew before the surgery, died from life-threatening complications during surgery, or were reassigned to alternative surgery were excluded. We enrolled patients with previous aortic valvuloplasty who ultimately received TAVI, and patients were eliminated if they failed to undergo TAVI after aortic valvuloplasty (Figure 1). All participants underwent a comprehensive preoperative assessment by a team of cardiologists. The final decision to perform TAVI or SAVR was based on a discussion between the cardiology team and the patient's relatives considering the optimal outcome in the best interests of the patient.

\section{Valve and procedure}

In the SAVR group, there were 16 mechanical heart valves and 93 bioprostheses, including the St. Jude Medical Epic heart valve (St. Jude Medical, St. Paul, MN, USA), St. Jude Medical Regent heart valve (St. Jude Medical, St. Paul, MN, USA), Perceval S (Sorin, Saluggia, Italy), Mosaic (Medtronic, Dublin, Ireland), Carpentier-Edwards Perimount Magna Ease (Edwards Lifesciences, Irvine, CA, USA), Hancock-II (Medtronic, Dublin, Ireland), and Open Pivot AP360 (Medtronic, Dublin, Ireland). The 5 different brands of TAVI prostheses examined in the current study were the Venus A-valve, J-Valve, TaurusOne, VitaFlow, and Sapien XT (Edwards Lifesciences, Irvine, CA, USA). All TAVI cases were handled by 2 cardiovascular surgeons proficient in cardiovascular intervention, and the diagnosis and treatment of cardiovascular diseases. The size and type of surgical valve were selected at the surgeon's discretion. Transthoracic echocardiography (TTE) and multi-detector 


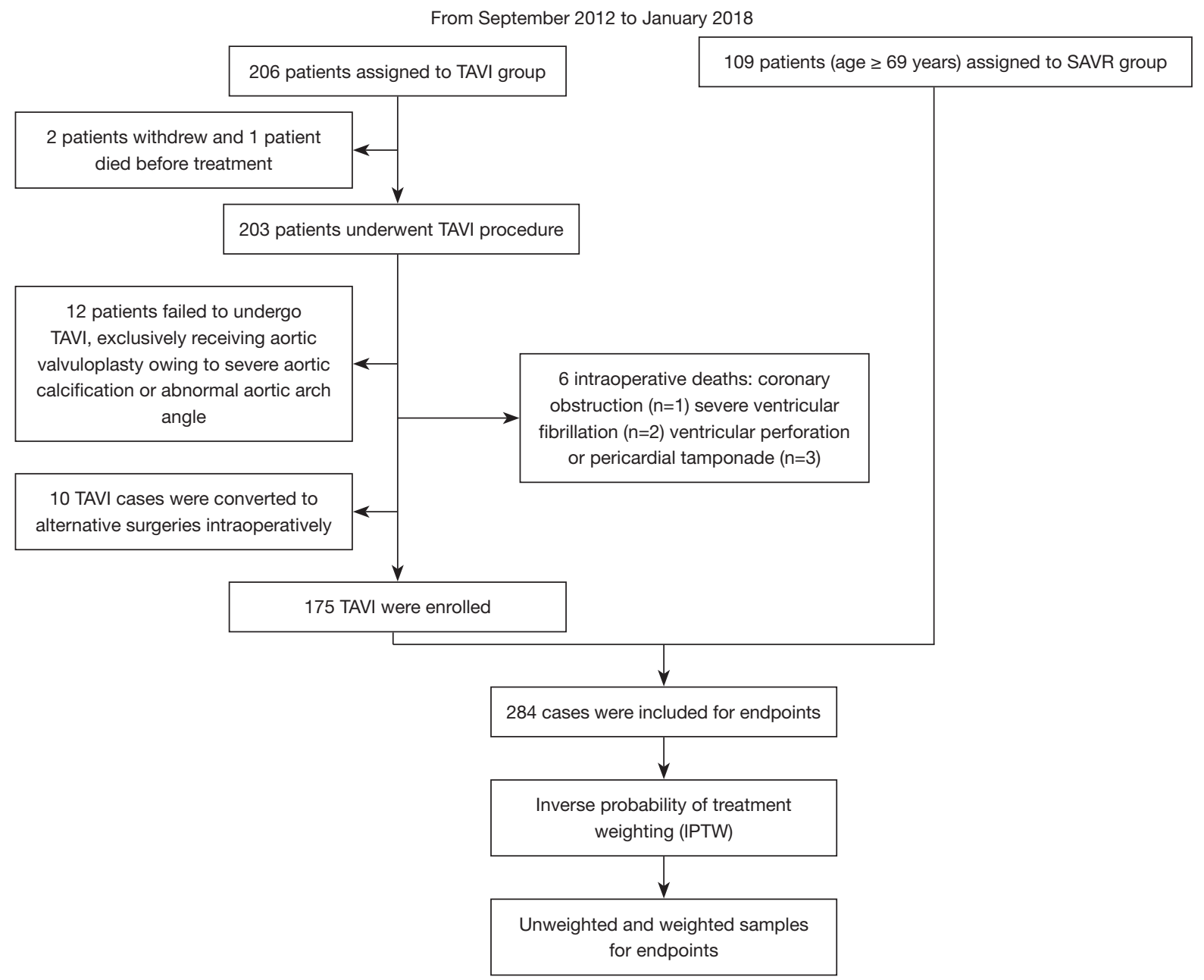

Figure 1 Flowchart of participant inclusion. TAVI, transcatheter aortic valve implantation; SAVR, surgical aortic valve replacement.

computed tomography (MDCT) were utilized for routine assessment of preoperative cardiac function, iliac vascular anatomy, valve morphology, and calcification in participants. Fluoroscopy, transesophageal echocardiography (TEE), or TTE was performed to confirm the appropriate placement of valves, and to evaluate the success of the procedure through the detection of valvular regurgitation, pleural effusion, and residual shunts. Vascular access was attained via the transfemoral, transapical, transaortic, or transcarotid routes, depending on the results of preprocedural peripheral vascular assessments. Notably, the J-Valve was received exclusively via a transapical approach.

\section{Endpoints}

Surgical outcomes and complications were defined in accordance with the Valve Academic Research Consortium-2 (VARC-2) (20). The primary endpoint was all-cause mortality. Secondary endpoints included stroke, new-onset atrial fibrillation (AF), permanent pacemaker implantation (PPI), myocardial infarction (MI), heart failure (HF), major vascular complication, high-grade atrioventricular block (HAVB), paravalvular leakage (PVL), and thrombosis. The operative duration was measured from the first incision to the final suture. Readmission unrelated to surgery or heart disease was excluded from the analysis, and death during readmission was not included in the analysis of readmission events. Intraoperative mortality was defined as death resulting from life-threatening events during the procedure. All-cause mortality was defined as the death of patients who survived surgery but died postoperatively. A composite of all-cause mortality, stroke, 
new-onset AF, PPI, MI, HF, and HAVB was used to evaluate the endpoints among the different valves.

Data were collected from electronic medical records at our institution. Information was also collected by telephone using a predefined questionnaire via follow-up system.

\section{Etbical statement}

This study adhered to the ethical principles of the Declaration of Helsinki (as revised in 2013). The ethics committee of Fuwai Hospital approved the retrospective collection of data (Approval No. 2020-1416), and the requirement for informed consent was waived due to the retrospective nature of the study.

\section{Statistical analysis}

The distribution of data was presented using descriptive statistics: mean \pm standard deviation (SD) or median [interquartile range (IQR)] was used for continuous variables, and absolute and relative frequencies were used for categorical variables. Continuous variables between groups were compared using the $t$-test or Mann-Whitney U-test based on the normality of distribution (Appendix 1). Categorical variables between groups were analyzed using the chi-square test. The Kruskal-Wallis test was applied to compare the postoperative length of hospital stay and operative duration among different valves. Kaplan-Meier survival analysis was used to describe the time to events, followed by the log-rank test comparing the endpoints between groups. Bonferroni correction was used for all pairwise comparisons among endpoints of valves.

To reduce potential bias, we introduced inverse probability of treatment weighting (IPTW) (21). IPTW weights individual patients on the basis of the inverse probability of their treatment allocation to create a pseudodata set in which the distribution of potentially confounding variables is balanced between the 2 groups. With this method, a logistic regression model was first fitted to generate a propensity score, adjusted for baseline covariates. Subsequently, the TAVI sample received a weight of $1 / p_{i}$, while the SAVR sample received a weight of $1 /\left(1-p_{i}\right)$, in which $p_{i}$ is individual $i$ s estimated propensity score (22). In the pseudo-dataset created by weighting each participant by IPTW, the number of observations is the sum of weights. The IPTW has the advantage of making use of all data. The literature has shown a trend toward the use of IPTW over the years (23).
The Cox proportional hazards model was then applied to analyze the predictors of all-cause mortality, postoperative stroke, and postoperative $\mathrm{HF}$ in both the weighted and unweighted samples. Variables with significance $(\mathrm{P}<0.1)$ upon univariate analysis or which were clinically relevant were included in a multivariate regression analysis to explore whether the endpoints could have been biased by baseline differences or outcomes. Appendix 1 shows the details of the univariate analyses and test of multicollinearity. The variables finally selected for inclusion in the model to predict all-cause mortality were liver disease, bicuspid valve, New York Heart Association (NYHA) class $\leq \mathrm{I}$, age, postoperative $\mathrm{HF}$, postoperative stroke, and TAVI. Variables in the HF prediction model included age, male sex, smoking history, ejection fraction, body mass index (BMI), TAVI, NYHA class $\leq \mathrm{II}$, diabetes, and hypertension. The variables enrolled in the stroke model were TAVI, age, MI, diabetes, stroke history, and hypertension. We conducted additional 10 models to test predictive validity of risk factors, which is shown in Appendix 1. Hazard ratio (HR) were also calculated to estimate the treatment effect between the 2 groups.

Tests of hypotheses were 2-sided and conducted at a standard statistical significance level of 0.05 , with a $95 \%$ confidence interval (CI) adopted. Statistical analyses were conducted using SPSS version 25 (IBM Corp., Armonk, NY, USA) and R version 4.0.2 (https://www.R-project.org/) with the "Survival", "reportReg", "MatchIt", "Survey", and "Tableone" packages.

\section{Results}

\section{General characteristics}

A total of 206 patients were scheduled for elective TAVI between September 2012 and January 2018. Prior to treatment, 2 patients withdrew and 1 died. Twelve participants exclusively underwent aortic valvuloplasty due to severe aortic calcification or an abnormal aortic arch angle. Ten TAVI cases were converted to alternative surgeries due to valve migration $(n=8)$, or severe ventricular fibrillation $(n=1)$ or cardiac tamponade $(n=1)$ before valve deployment. Six participants died intraoperatively. The major causes of death included coronary obstruction $(n=1)$, severe ventricular fibrillation $(n=2)$, and ventricular perforation or pericardial tamponade $(n=3)$. Ultimately, 175 participants received TAVI successfully (Figure 1). Among them, 9 were treated with Sapien XT, 97 with Venus A, 17 with TaurusOne, 25 with VitaFlow, and 27 with J-Valve. 
Table 1 Baseline characteristics

\begin{tabular}{|c|c|c|c|c|c|c|}
\hline Baseline & \multicolumn{3}{|c|}{ Overall Cohort } & \multicolumn{3}{|c|}{ IPTW Matched Cohort } \\
\hline Age, years & $72.72 \pm 2.69$ & $76.57 \pm 5.84$ & $<0.001^{*}$ & $74.79 \pm 3.79$ & $74.55 \pm 7.19$ & 0.861 \\
\hline Male, n (\%) & $65(59.6)$ & $104(59.4)$ & 0.973 & $(74.8)$ & $(63.5)$ & 0.161 \\
\hline $\mathrm{BMI}, \mathrm{kg} / \mathrm{m}^{2}$ & $24.68 \pm 3.14$ & $23.17 \pm 3.72$ & $0.001^{*}$ & $22.91 \pm 3.92$ & $23.80 \pm 3.69$ & 0.381 \\
\hline Hypertension, n (\%) & $52(47.7)$ & $109(62.3)$ & $0.016^{\star}$ & $(56.8)$ & $(53.9)$ & 0.798 \\
\hline Diabetes, n (\%) & $14(12.8)$ & $52(29.7)$ & $0.001^{*}$ & $(11.2)$ & $(24.2)$ & 0.068 \\
\hline Liver disease, $\mathrm{n}(\%)$ & $0(0.0)$ & $4(2.3)$ & 0.284 & $(0.0)$ & $(1.5)$ & 0.057 \\
\hline Syncope, n (\%) & $20(18.3)$ & $32(18.3)$ & 0.989 & $(17.3)$ & $(17.0)$ & 0.958 \\
\hline PCl, n (\%) & $3(2.8)$ & $28(16.0)$ & $<0.001^{*}$ & $(8.4)$ & $(11.6)$ & 0.638 \\
\hline Peripheral vascular disease, $\mathrm{n}(\%)$ & $20(18.3)$ & $52(29.7)$ & $0.032^{*}$ & $(16.5)$ & $(24.9)$ & 0.280 \\
\hline Stroke history, n (\%) & $25(22.9)$ & $100(57.1)$ & $<0.001^{\star}$ & $(41.8)$ & $(47.3)$ & 0.656 \\
\hline HAVB, n (\%) & $0(0.0)$ & $4(2.3)$ & 0.284 & $(0.0)$ & (1.5) & 0.057 \\
\hline Atrial fibrillation, n (\%) & $7(6.4)$ & $39(22.3)$ & $<0.001^{*}$ & $(6.6)$ & $(16.2)$ & 0.053 \\
\hline Inotropic drugs, n (\%) & $11(10.1)$ & $35(20.0)$ & $0.028^{*}$ & $(22.4)$ & $(19.5)$ & 0.815 \\
\hline ACEI/ARB drugs within 48hours, $n(\%)$ & $15(13.8)$ & $42(24.0)$ & $0.036^{\star}$ & $(31.0)$ & $(21.1)$ & 0.390 \\
\hline $\mathrm{EF}, \mathrm{n}(\%)$ & $60.53 \pm 8.01$ & $57.10 \pm 12.50$ & $0.011^{*}$ & $57.35 \pm 8.41$ & $57.42 \pm 12.33$ & 0.977 \\
\hline CLBBB, n (\%) & $2(1.8)$ & $8(4.6)$ & 0.376 & (3.5) & (3.9) & 0.907 \\
\hline Pure aortic regurgitation, $\mathrm{n}(\%)$ & $5(4.6)$ & $5(2.9)$ & 0.661 & $(3.4)$ & $(4.0)$ & 0.841 \\
\hline Smoking history, n (\%) & $34(31.2)$ & $66(37.7)$ & 0.263 & $(53.2)$ & (39.9) & 0.237 \\
\hline
\end{tabular}

Values are mean $\pm \mathrm{SD}$, median $(\mathrm{IQR})$ or $\mathrm{n}(\%) .{ }^{*}$, significant values. ${ }^{\dagger}, \mathrm{n}$ is the weighted sample induced by IPTW. ACEI, angiotensin converting enzyme inhibitors; ARB, angiotensin receptor blocker; BMI, body mass index; CLBBB, complete left bundle branch block; COPD, chronic obstructive pulmonary disease; CRBBB, complete right bundle branch block; EF, ejection fraction; HAVB, high-grade atrioventricular block; IPTW, inverse probability of treatment weighting; NYHA, New York heart association; PLT, platelet; PCI, percutaneous coronary intervention; SAVR, surgical aortic valve replacement; STS, society of thoracic surgeons; TAVI, transcatheter aortic valve implantation.

Two participants who had been assigned to transfemoral TAVI underwent conversion to transaortic access intraprocedurally. Finally, in the TAVI group, transfemoral access was used in 134 cases, transapical access in 26 cases, transaortic access in 13 cases, and transcarotid access in 2 cases. During the same period, 109 patients undergoing SAVR were enrolled, none of whom died intraoperatively. As shown in Table 1, there were significant differences between the groups at baseline. However, after 
adjustment by IPTW, no statistical differences were observed.

\section{Postoperative outcomes}

Follow-up was performed for a median of 29 months (IQR: 16.5, 46 months). Participants in the TAVI group were less likely to require general anesthesia (38.9\% vs. $100 \%$, $\mathrm{P}<0.001)$ and intensive care unit (ICU) admission $(37.1 \%$ vs. $100 \%, \mathrm{P}<0.001)$ than patients in the SAVR group, and the TAVI group also had a shorter operative duration (115 vs. 184, $\mathrm{P}<0.001)$. These outcomes were robust after IPTW adjustment (IPTW-adjusted $\mathrm{P}<0.001$ ). The TAVI cohort also appeared to have higher rates of all-cause mortality (16.3\% vs. $4.4 \%$, TAVI vs. SAVR, HR: $3.79,95 \%$ CI: 1.31 to $10.96, \mathrm{P}=0.014)$ and PPI $(17.0 \%$ vs. $2.9 \%$, TAVI vs. SAVR, HR: $6.17,95 \% \mathrm{CI}: 1.87$ to $20.36, \mathrm{P}=0.003)$ at 3 years than the SAVR cohort. These results were in line with the effects after IPTW adjustment (all-cause mortality: $11.4 \%$ vs. $2.4 \%$, HR: $4.79,95 \%$ CI: 1.47 to 15.57 , IPTWadjusted $\mathrm{P}=0.009$; PPI: $14.6 \%$ vs. $1.6 \%$, HR: $9.98,95 \% \mathrm{CI}$ : 2.71 to 36.67 , TAVI $v$ s. SAVR, IPTW-adjusted $\mathrm{P}<0.001$ ). With regard to postoperative HF, the TAVI cohort had a higher rate of $\mathrm{HF}(7.2 \%$ vs. $1.8 \%$, TAVI $v$ s. SAVR, HR: $3.57,95 \%$ CI: 0.79 to $16.1 \mathrm{P}=0.098$ ) at 3 years than the SAVR cohort, although there was no statistical difference. However, the difference became slightly significant after IPTW adjustment ( $4.9 \%$ vs. $0.9 \%$, TAVI vs. SAVR, HR: 4.89, 95\% CI: 1.0 to 23.95, IPTW-adjusted $\mathrm{P}=0.051)$. The differences in $\mathrm{AF}$ and readmission at 3 years between the TAVI and SAVR cohorts were not statistically significant, and remained insignificant after IPTW adjustment (Table 2).

Kaplan-Meier curves estimating the mid- to longterm survival of the 2 groups are shown in Figure 2. The cumulative all-cause mortality showed a trend toward a higher incidence in the TAVI group than in the SAVR group (HR: 5.49, 95\% CI: 1.71 to 17.68 , IPTW-adjusted P=0.004).

\section{Causes of mortality and readmission}

After discharge, 24 participants were readmitted to the hospital and 26 died on the basis of the available data. The causes of readmission and death were analyzed individually and are identified in Figure 3. The leading causes of readmission in the TAVI group were HF (25\%) and stroke (20.8\%).

\section{Predictors of poor outcomes}

Multivariable Cox regression was used to analyze the predictors of all-cause mortality, stroke, and HF. The adjusted results revealed that liver disease was a risk predictor of all-cause mortality (HR: 5.080, 95\% CI: 1.067 to $24.174, \mathrm{P}=0.041$ ). After IPTW adjustment, the effect remained (HR: $8.421,95 \%$ CI: 1.813 to 39.108 , IPTWadjusted $\mathrm{P}=0.007)$. A history of smoking was related to the occurrence of postoperative HF (HR: 4.902, 95\% CI: 1.265 to $18.999, \mathrm{P}=0.022$ ) and this effect remained after IPTW adjustment. Additionally, age (HR: 1.141, 95\% CI: 1.010 to 1.288, $\mathrm{P}=0.034$ ) and diabetes (HR: 7.301, 95\% CI: 2.414 to $22.079, \mathrm{P}<0.001)$ were found to be related to the increased risk of postoperative stroke in the multivariable Cox regression; however, these effects were slightly attenuated after IPTW adjustment (age: IPTW-adjusted $\mathrm{P}=0.051$, diabetes: IPTW-adjusted $\mathrm{P}=0.082$ ) (Figure 4).

\section{Valve comparison}

We compared the outcomes of TAVI procedures performed using Venus A, TaurusOne, VitaFlow, and J-Valve. With the exception of readmission ( $\mathrm{P}=0.038)$, the 1 -year outcomes did not differ significantly between the valves. Specifically, TaurusOne (23.5\%) had the highest occurrence of readmission, followed by VitaFlow (4.5\%), Venus A (4.4\%), and J-Valve (4.3\%). The all-cause mortality rate was the highest among participants who received J-Valve (19.0\%); However, the difference was not significant. Participants who received TaurusOne had a briefer postoperative length of stay (7 days) compared with those who received Venus A (9 days, adjusted $\mathrm{P}=0.003$ ), VitaFlow ( 8 days, adjusted $\mathrm{P}=0.006$ ), and $\mathrm{J}$-Valve (10 days, adjusted $\mathrm{P}=0.001)$. The composite endpoints in the VitaFlow subgroup remained the lowest (28\%) compared with those of Venus A (38.2\%), TaurusOne (35.3\%), VitaFlow (28\%), and J-Valve (34\%), but there was no statistical difference $(\mathrm{P}=0.896)$ (Table 3).

We ran Cox regression separately to explore whether the type of valve used had an impact on all-cause mortality, stroke, postoperative HF, and readmission. The results revealed that none of the valves had a significant association with all-cause mortality, postoperative stroke, or HF (Table S1).

\section{$P V L$}

The incidence of PVL was calculated independently based on the data available. The detailed data available from follow-up are shown in Table S2. The rate of $>$ mild PVL (moderate or severe PVL) in the TAVI cohort at 
Table 2 Postoperative outcomes

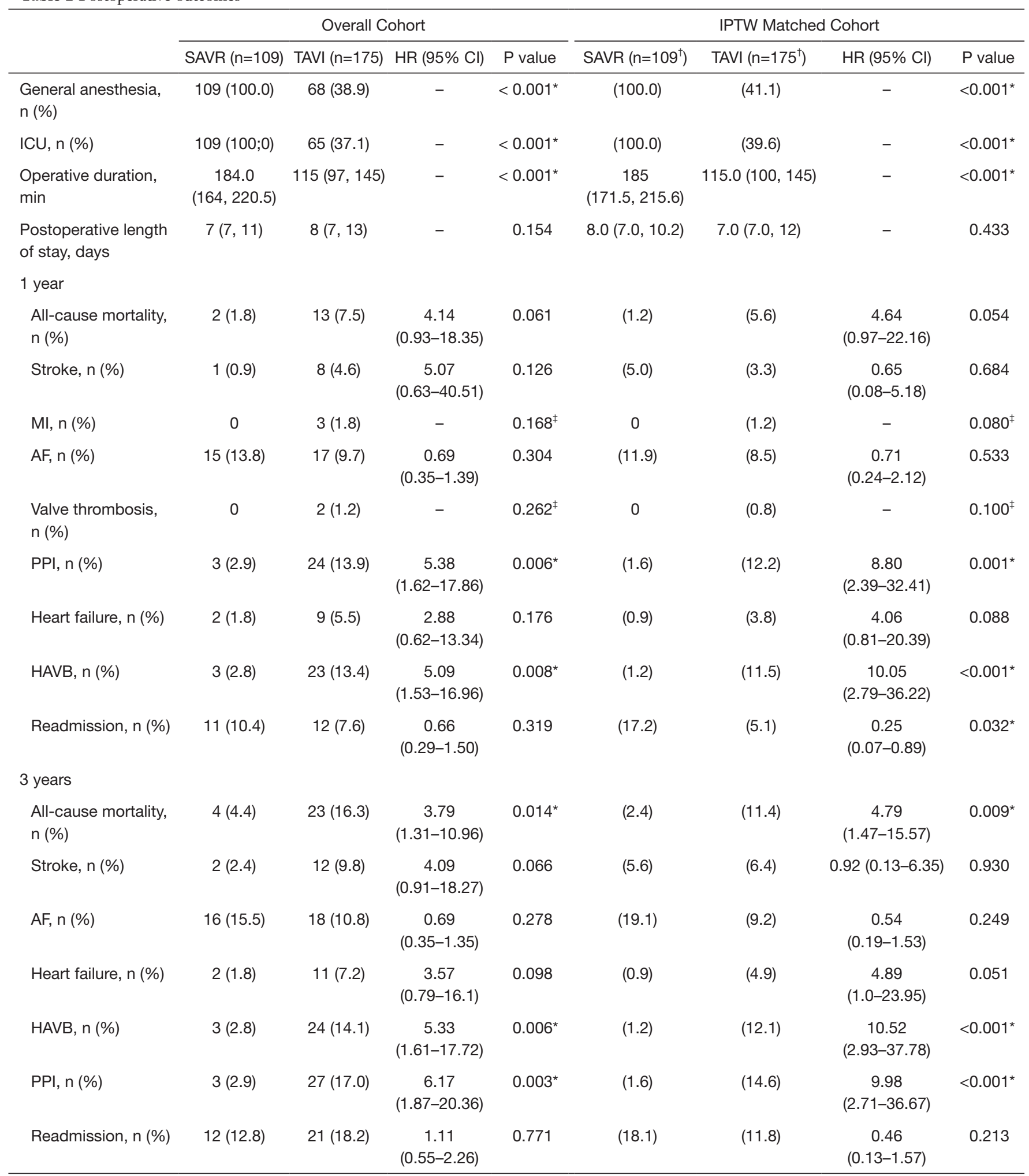

Values are median (IQR) or $\mathrm{n}(\%) .{ }^{*}$, significant values. ${ }^{\ddagger}$, values from log rank test. ${ }^{\dagger}, \mathrm{n}$ is the weighted sample induced by IPTW. AF, atrial fibrillation; Cl, confidence interval; HAVB, high-grade atrioventricular block; HR, hazard ratio; ICU, intensive care unit; MI, myocardial infarction; PPI, permanent pacemaker implantation. SAVR, surgical aortic valve replacement; TAVI, transcatheter aortic valve implantation. 

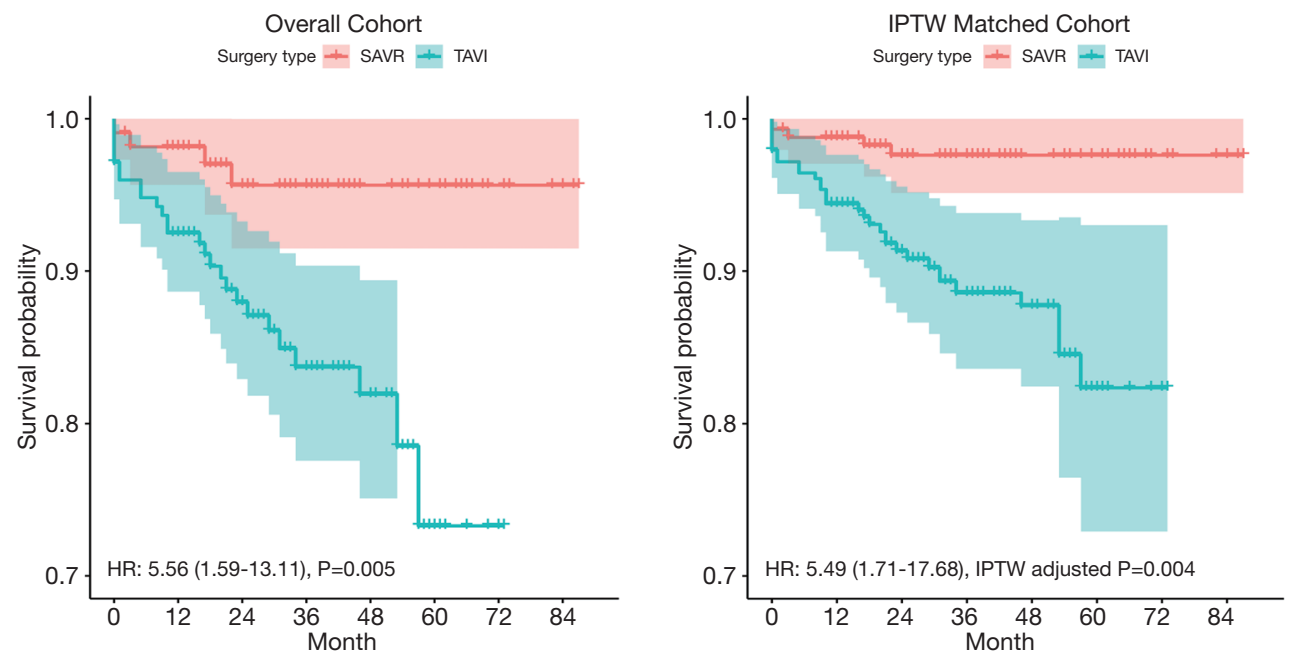

Figure 2 Kaplan-Meier curves estimating mid-term survival. TAVI, transcatheter aortic valve implantation; SAVR, surgical aortic valve replacement; IPTW, inverse probability of treatment weighting; HR, hazard ratio
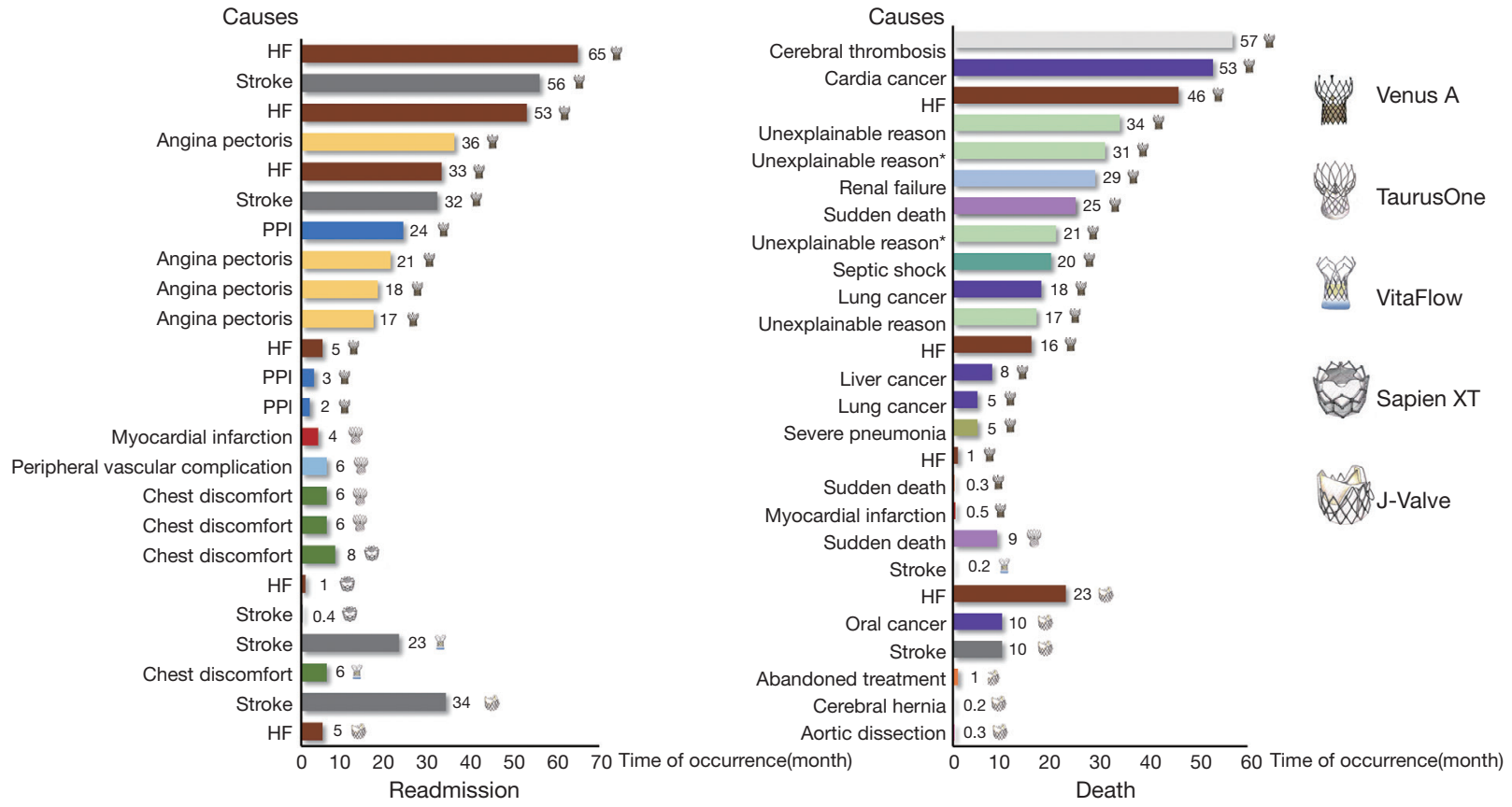

Figure 3 Causes of mortality and readmission in the TAVI group. *, suspected cardiac causes. HF, heart failure; TAVI, transcatheter aortic valve implantation; PPI, permanent pacemaker implantation.

1 week, 1 month, and 1 year after surgery was $6.6 \%$, $5.5 \%$, and $2.6 \%$, respectively. Compared with SAVR (following which no cases of mild, moderate, or severe PVL were reported), TAVI was still inferior $(\mathrm{P}<0.001)$. The rates of $\geq$ mild PVL (mild or worse PVL) among participants who received Sapien XT and Venus A were similar, and were higher than those for TaurusOne, J-Valve, and VitaFlow. Notably, for VitaFlow, no cases of $>$ mild PVL were detected. However, the differences were not significant (Figure 5, Table S2). 


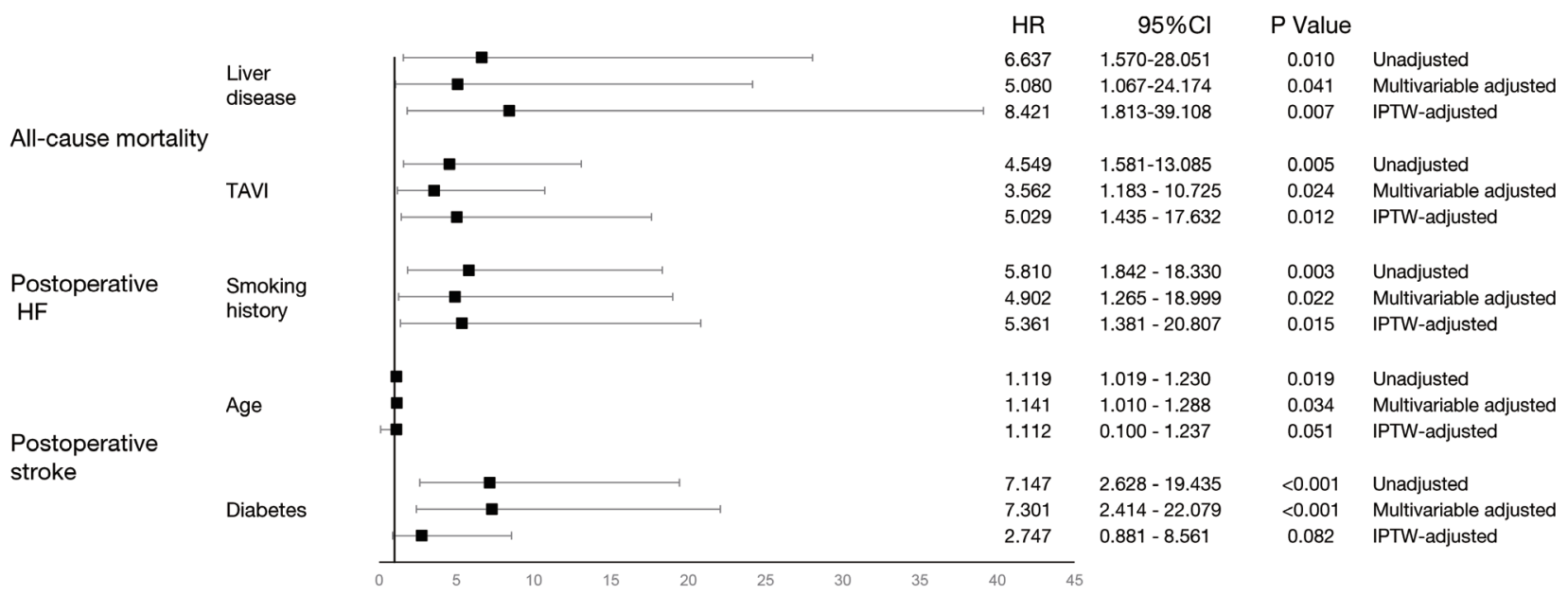

Figure 4 Predictors of poor outcomes in Cox regression. CI, confidence interval; HF, heart failure; HR, hazard ratio; TAVI, transcatheter aortic valve implantation.

Table 3 Perioperative and 1-year outcomes among different valves

\begin{tabular}{|c|c|c|c|c|c|c|c|}
\hline Outcomes & Venus $A(n=97)$ & TaurusOne $(n=17)$ & VitaFlow $(n=25)$ & J-Valve $(n=27)$ & $\mathrm{P}^{\S}$ value & Sapien XT $(n=9)$ & SAVR $(n=109)$ \\
\hline \multicolumn{8}{|l|}{ Perioperative Outcomes } \\
\hline Reintervention, n (\%) & $11(11.3)$ & $3(17.6)$ & $4(16.0)$ & $1(3.7)$ & 0.378 & 0 & - \\
\hline Valve-in-valve, n (\%) & $11(11.3)$ & $2(11.8)$ & $2(8.0)$ & 0 & 0.276 & 0 & - \\
\hline General anesthesia, n (\%) & $26(26.8)$ & $6(35.3)$ & $3(12.0)$ & $27(100.0)$ & $<0.001^{*}$ & $6(66.7)$ & $109(100.0)$ \\
\hline $\begin{array}{l}\text { Postoperative length of } \\
\text { stay, days }\end{array}$ & $9(7,13)$ & $7(5,7)$ & $8(7,13.5)$ & $10(7,14)$ & $0.001^{*}$ & $7.0(7,7.5)$ & $7(7,11)$ \\
\hline \multicolumn{8}{|l|}{ 1-year outcomes, n (\%) } \\
\hline $\begin{array}{l}\text { Major vascular } \\
\text { complication }\end{array}$ & $5(5.2)$ & $1(5.9)$ & 0 & $1(3.7)$ & 0.700 & 0 & $1(1.0)$ \\
\hline Stroke & $5(5.2)$ & 0 & $1(4.0)$ & $1(3.7)$ & 0.807 & $1(11.1)$ & $1(0.9)$ \\
\hline $\mathrm{AF}$ & $7(7.2)$ & $3(17.6)$ & $3(12.0)$ & $2(7.4)$ & 0.519 & $2(22.2)$ & $15(13.8)$ \\
\hline Heart failure & $6(6.5)$ & 0 & $1(4.0)$ & $1(4.3)$ & 0.707 & 1 (11.1) & $2(1.8)$ \\
\hline MI & $2(2.1)$ & $1(5.9)$ & 0 & 0 & 0.490 & 0 & 0 \\
\hline HAVB & 16 (16.9) & $1(5.9)$ & $4(16.0)$ & $1(3.7)$ & 0.266 & $1(11.1)$ & $3(2.8)$ \\
\hline PPI & $16(16.8)$ & $2(11.8)$ & $4(16.0)$ & $1(3.7)$ & 0.393 & $1(11.1)$ & $3(2.9)$ \\
\hline
\end{tabular}

Values are median (IQR) or $\mathrm{n}(\%)$. *significant values. $\mathrm{P}^{\S}$ value was the difference among Venus A, TaurusOne, VitaFlow, and J-Vale. AF, atrial fibrillation; HAVB, high-grade atrioventricular block; HR, hazard ratio; ICU, intensive care unit; MI, myocardial infarction; PPI, permanent pacemaker implantation. SAVR, surgical aortic valve replacement. 


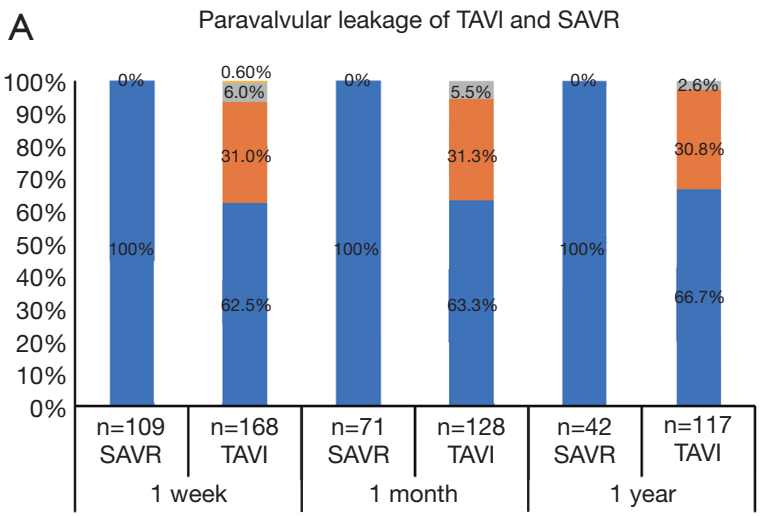

- None/trace $\mid$ " Mild $\mid$ | Moderate $\mid$ Severe

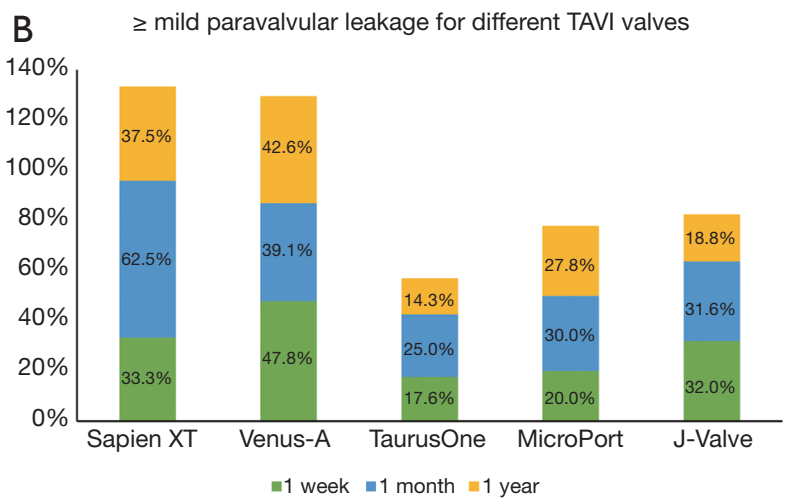

Figure 5 Paravalvular leakage at 1 year. (A) Paravalvular leakage after TAVI and SAVR, and (B) $\geq$ mild paravalvular leakage for different TAVI valves. TAVI, transcatheter aortic valve implantation; SAVR, surgical aortic valve replacement.

\section{Discussion}

We defined "early TAVI" as a procedure performed in the 6 years after TAVI was developed at our institution. During this period, the number of TAVI procedures was limited, averaging approximately 40 procedures per year. It was not until 2018 that TAVI began to exceed 100 cases per year (unpublished data). The results of the present study showed that TAVI was associated with increased all-cause mortality compared with SAVR, with or without IPTW adjustment, which appears to contradict previous reports $(24,25)$. It could be assumed that the poor outcomes may have resulted from the lack of experience; therefore, we divided TAVI cases into 4 stages according to time and compared the outcomes of every stage to explore the impact of experience on outcomes. The results did not vary widely between the "early" and "recent" stages (Table S3, Figure S1). Moreover, 2 surgeons (including an interventional cardiologist), both of whom are proficient in the field, conducted all TAVI procedures and sometimes collaborated to complete TAVI. Accordingly, it was less likely that the variance between surgeons or the lack of experience contributed significantly to poor outcomes. Also, we included patients with pure aortic regurgitation, which might be associated with an inferior outcome (26). Accordingly, we conducted a sensitivity analysis to examine whether aortic regurgitation had influenced outcomes. The results revealed that aortic regurgitation barely impacted patients' prognoses (Table S4). Nevertheless, the reasons for the poor outcomes of patients could be complex. Furthermore, whether or not the outcomes were related to the characteristics of the Asian study population remains unclear. Further studies to compare the outcomes between populations from Asia and other continents are needed to elucidate this issue.

This study, to some extent, has outlined 1 reason why TAVI has developed slowly in Asia: surgeons are prudent in the selection of recipients, and also consider patients' preferences. However, due to this study being restricted by its sample size and retrospective characteristics, it has limited power to elucidate this vital issue. Accordingly, future randomized trials comparing TAVI with SAVR in Asia will be of paramount importance. Currently, TAVI has showed a trend toward being increasingly adopted worldwide due to its minimally invasive nature, and we are not able to reject the expansion of this technique in Asia merely on the grounds of the current outcomes. Instead, more investigations should be conducted to explore the underlying factors that have precluded the rapid expansion of TAVI in Asia. For centers that initiate TAVI, effective communication with patients, sufficient preparation, proper perioperative management, and immediate summaries of experience are of paramount importance to avoiding poor outcomes.

Postoperative HF was the leading cause of readmission (25\%) and a predictor of death (HR: 2.985, 95\% CI: 1.068 to 8.340 , adjusted $\mathrm{P}=0.037$ ) among patients in the study (Appendix 1). Similar results were observed in a study by Saji et al., in which $25 \%$ of patients had unplanned readmission following TAVI, mainly due to HF (27). Patients who receive a prescription for a renin-angiotensin system inhibitor at hospital discharge have been found to have a significantly lower risk of mortality and HF readmission than those who receive no prescription (28). The underlying risk factors for HF include chronic pulmonary and kidney disease, diabetes mellitus, previous AF, and a post-TAVI left ventricular ejection fraction of $\leq 35 \%$ (29). Smoking has previously been estimated to be responsible for $17 \%$ of $\mathrm{HF}$ cases $(30,31)$, and we also found 
that a smoking history carried significant prognostic value, with a strong association with HF. Additionally, Aune et al. concluded that the HF risk decreases as the time since smoking cessation lengthens (32). Great variation exists between reports regarding the smoking cessation duration required to achieve improved outcomes; however, it is widely accepted that the longer the duration of cessation preoperatively, the greater the benefit to the patient (33).

Stroke was the second leading cause of readmission in this study and has been reported to be correlated with a significant increase in 30-day mortality (HR: 6.1, 95\% CI: 5.4 to $6.8, \mathrm{P}<0.001$ ) (34). The rate of postoperative stroke $(9.8 \%$ vs. $2.4 \%$, HR: 4.09 , $95 \%$ CI: 0.91 to 18.27 , $\mathrm{P}=0.066$ ) was slightly higher in the TAVI group than that in the SAVR group in our center; this result contradicts the findings of previous studies $(1,35,36)$, and was probably influenced by preoperative confounders. However, this effect was obviously attenuated after IPTW adjustment (6.4\% vs. 5.6\%, HR: 0.92, 95\% CI: 0.13 to 6.35, TAVI vs. SAVR, IPTW-adjusted $\mathrm{P}=0.93)$. Moreover, recent research has shown that TAVI resulted in a lower risk of stroke (HR: 0.81, 95\% CI: 0.68 to $0.98, \mathrm{P}=0.028$ ) than did SAVR (24). Cox regression analysis further revealed that while stroke was not related to the type of surgery, it was associated with age and diabetes. Older patients with diabetes are more likely to suffer from vascular lesions that increase the risk of stroke. Accordingly, stroke might not preclude the expansion of TAVI; however, identifying risk factors, optimal monitoring, and immediate medical treatment intensification are all paramount to improving the quality of care and reducing the healthcare costs associated with this treatment.

To the best of our knowledge, this study has provided a preliminary comparison of the newly developed valves, which will help to fill in the gap in valve selection and improvement. Notably, patients who received J-Valve were more likely to require general anesthesia and ICU admission $(\mathrm{P}<0.001)$. These differences were mainly associated with the type of access, with TAVI via the transapical approach (for J-Valve) being performed under general anesthesia and requiring routine transfer to the ICU. It should be emphasized that Sapien XT accounted for the highest rates of readmissions $(33.3 \%)$. Also, the rate of $\geq$ mild PVL in Sapien XT cases varied widely (33.3-62.5\%), though it has been observed as $54.3 \%$ elsewhere (37). To a large extent, this finding was attributable to the small sample size of Sapien XT cases $(n=9)$. To date, the causes of readmission at our hospital have varied widely between the valves (Figure 3).
Further studies with a larger sample size in this TAVI group will better elucidate this issue.

Overall, VitaFlow was associated with the lowest composite endpoint and PVL (with no cases of $>$ mild PVL detected in the available data), although there were no statistical differences (Table S2), and there were no other notable outcomes. On the basis of a comprehensive assessment, among the valves in question, VitaFlow may be associated with slightly superior outcomes; however, conclusive evidence is lacking. Further comparisons between these valves should be performed through randomized studies or well-controlled prospective registries.

\section{Limitations}

First, as this was a retrospective, single-center, nonrandomized controlled trial, there were several potential unmeasured variables that may have influenced outcomes, such as frailty and aortic valve calcium score. This study was limited by its small sample size and the research characteristics; thus, further prospective studies with a large sample size will be necessary to verify our results. Second, identification of PVL relied on echocardiographic findings in Fuwai Hospital. Since some participants, particularly those with SAVR, returned to local hospitals for further examination, which made their PVL data difficult to obtain. Furthermore, 6 participants died intraoperatively, and the current study did not investigate the details of those cases. Additionally, only 9 participants received Sapien $\mathrm{XT}$, and this insufficient number resulted in the failure to make any comparative conclusions regarding this valve. Finally, following IPTW, the model might not have been well established, which to some extent contributed to the underlying outcome bias. Owing to the limited sample size, the vital HR value in our study had a wide $95 \%$ CI.

\section{Conclusions}

In the first 6 years in which we initiated TAVI at our hospital, TAVI was mainly applied to specific patients. We found that TAVI is associated with higher rates of all-cause mortality, PPI, HF, and PVL than SAVR; however, it has lower rates of general anesthesia use and ICU admission, as well as a shorter operative duration. Liver disease was identified as a predictor of all-cause mortality, and a smoking history was observed to increase the risk of HF. Age and diabetes increase the risk of postoperative stroke. The VitaFlow valve appears to have the lowest composite 
endpoint among the new Chinese valves examined, although there is an absence of conclusive evidence.

Our results have demonstrated that not all TAVI procedures attained satisfactory outcomes compared with SAVR when initiated. At first, our center faced some challenges in delivering TAVI, and this is probably 1 of the reasons why the adoption of TAVI has been slow in Asia. Further investigations are needed to explore the underlying factors which have precluded the rapid expansion of TAVI in Asia.

\section{Acknowledgments}

The authors would like to extend their gratitude to Jingxin Yao at Tiantan Hospital for her work in collecting part of the data, and to Can Cai, Key Laboratory of Cardiovascular Epidemiology and Department of Epidemiology, for her support and help with statistics. The authors also thank the editor J. Jones and J. Reynolds in AME Editing Service, for their English editing help. The authors thank Runyu Zhang, BEIJING ETOP LAW FIRM, for his legal advice about copyright. Finally, the authors thank Hui Gong and Jie Gao, for her artistical guidance of the figure.

Funding: This study is supported by National Natural Science Foundation of China (81670077), Innovative Research Foundation of Peking Union Medical College (2019-1002-77), CAMS Innovation Fund for Medical Sciences (2020-I2M-C\&T-B-066).

\section{Footnote}

Reporting Checklist: The authors have completed the STROBE reporting checklist. Available at https://dx.doi. org/10.21037/cdt-20-928

Data Sharing Statement: Available at https://dx.doi. org/10.21037/cdt-20-928

Conflicts of Interest: All authors have completed the ICMJE uniform disclosure form (available at https://dx.doi. org/10.21037/cdt-20-928). The authors have no conflicts of interest to declare

Ethical Statement: The authors are accountable for all aspects of the work in ensuring that questions related to the accuracy or integrity of any part of the work are appropriately investigated and resolved. This study was conducted in accordance with the Declaration of Helsinki (as revised in 2013). The study was approved by Ethics Committee of
Fuwai Hospital (Approval No. 2020-1416) and individual consent for this retrospective analysis was waived.

Open Access Statement: This is an Open Access article distributed in accordance with the Creative Commons Attribution-NonCommercial-NoDerivs 4.0 International License (CC BY-NC-ND 4.0), which permits the noncommercial replication and distribution of the article with the strict proviso that no changes or edits are made and the original work is properly cited (including links to both the formal publication through the relevant DOI and the license). See: https://creativecommons.org/licenses/by-nc-nd/4.0/.

\section{References}

1. Mack MJ, Leon MB, Thourani VH, et al. Transcatheter Aortic-Valve Replacement with a Balloon-Expandable Valve in Low-Risk Patients. N Engl J Med 2019;380:1695-705.

2. Popma JJ, Deeb GM, Yakubov SJ, et al. Transcatheter Aortic-Valve Replacement with a Self-Expanding Valve in Low-Risk Patients. N Engl J Med 2019;380:1706-15.

3. Gomez CA, Braghiroli J, de Marchena E. "The changing paradigm": TAVR for low-risk patients approved by the FDA. J Card Surg 2020;35:5-7.

4. Hon JKF, Tay E. Transcatheter aortic valve implantation in Asia. Ann Cardiothorac Surg 2017;6:504-9.

5. Chiam PT, Koh TH, Chao VT, et al. Percutaneous transcatheter aortic valve replacement: first transfemoral implant in Asia. Singapore Med J 2009;50:534-7.

6. Chiam PTL. Transcatheter aortic valve implantation in Asia: the first decade. EuroIntervention 2018;14:35-7.

7. Giannini F, Baldetti L, Gallone G, et al. Transcatheter Valve Replacement in Asia Pacific: Current Practice and Perspectives. J Am Coll Cardiol 2018;72:3189-99.

8. Yoon SH, Ahn JM, Hayashida K, et al. Clinical Outcomes Following Transcatheter Aortic Valve Replacement in Asian Population. JACC Cardiovasc Interv 2016;9:926-33.

9. Kong WKF, Regeer MV, Poh KK, et al. Inter-ethnic differences in valve morphology, valvular dysfunction, and aortopathy between Asian and European patients with bicuspid aortic valve. Eur Heart J 2018;39:1308-13.

10. Inohara T, Vemulapalli S, Kohsaka S, et al. Appropriateness of Transcatheter Aortic Valve Replacement: Insight From the OCEAN-TAVI Registry. Circ Cardiovasc Qual Outcomes 2020;13:e006146.

11. Yoon SH, Ohno Y, Araki M, et al. Comparison of aortic root anatomy and calcification distribution between Asian and Caucasian patients who underwent transcatheter aortic 
valve implantation. Am J Cardiol 2015;116:1566-73.

12. Yu CW, Kim WJ, Ahn JM, et al. Trends and Outcomes of Transcatheter Aortic Valve Implantation (TAVI) in Korea: the Results of the First Cohort of Korean TAVI Registry. Korean Circ J 2018;48:382-94.

13. Venus MedTech. Accessed 22th May 2021. Available online: http://www.venusmedtech.com/

14. Suzhou JiechengMedical Technology. Accessed 22th May 2021. Available online: http://en.jiechengmedical.com

15. Peijia Medical. Accessed 22th May 2021. Available online: https://www.peijiamedical.com

16. MicroPort. Accessed 22th May 2021. Available online: http://www.cardioflowmedtech.com

17. Zhou D, Pan W, Wang J, et al. VitaFlow ${ }^{\mathrm{TM}}$ transcatheter valve system in the treatment of severe aortic stenosis: One-year results of a multicenter study. Catheter Cardiovasc Interv 2020;95:332-8.

18. Liao YB, Zhao ZG, Wei X, et al. Transcatheter aortic valve implantation with the self-expandable venus A-Valve and CoreValve devices: Preliminary Experiences in China. Catheter Cardiovasc Interv 2017;89:528-33.

19. Luo X, Wang X, Li X, et al. Transapical transcatheter aortic valve implantation using the J-Valve system: A 1-year follow-up study. J Thorac Cardiovasc Surg 2017;154:46-55.

20. Kappetein AP, Head SJ, Généreux P, et al. Updated standardized endpoint definitions for transcatheter aortic valve implantation: the Valve Academic Research Consortium-2 consensus document (VARC-2). Eur J Cardiothorac Surg 2012;42:S45-60.

21. Austin PC, Stuart EA. Moving towards best practice when using inverse probability of treatment weighting (IPTW) using the propensity score to estimate causal treatment effects in observational studies. Stat Med 2015;34:3661-79.

22. Lee BK, Lessler J, Stuart EA. Improving propensity score weighting using machine learning. Stat Med 2010;29:337-46.

23. Jochheim D, Barbanti M, Capretti G, et al. Oral Anticoagulant Type and Outcomes After Transcatheter Aortic Valve Replacement. JACC Cardiovasc Interv 2019;12:1566-76.

24. Siontis GCM, Overtchouk P, Cahill TJ, et al. Transcatheter aortic valve implantation vs. surgical aortic valve replacement for treatment of symptomatic severe aortic stenosis: an updated meta-analysis. Eur Heart J 2019;40:3143-53.

25. Arora S, Vaidya SR, Strassle PD, et al. Meta-analysis of transfemoral TAVR versus surgical aortic valve replacement. Catheter Cardiovasc Interv 2018;91:806-12.

26. Yousef A, MacDonald Z, Simard T, et al. Transcatheter Aortic Valve Implantation (TAVI) for Native Aortic Valve Regurgitation - A Systematic Review. Circ J 2018;82:895-902.
27. Saji M, Higuchi R, Tobaru T, et al. Impact of Frailty Markers for Unplanned Hospital Readmission Following Transcatheter Aortic Valve Implantation. Circ J 2018;82:2191-8.

28. Inohara T, Manandhar P, Kosinski AS, et al. Association of Renin-Angiotensin Inhibitor Treatment With Mortality and Heart Failure Readmission in Patients With Transcatheter Aortic Valve Replacement. JAMA 2018;320:2231-41.

29. Guedeney P, Huchet F, Manigold T, et al. Incidence of, risk factors for and impact of readmission for heart failure after successful transcatheter aortic valve implantation. Arch Cardiovasc Dis 2019;112:765-72.

30. He J, Ogden LG, Bazzano LA, et al. Risk factors for congestive heart failure in US men and women: NHANES I epidemiologic follow-up study. Arch Intern Med 2001;161:996-1002.

31. Prescott E. Smoking and heart failure: A call for action. Eur J Prev Cardiol 2019;26:277-8.

32. Aune D, Schlesinger S, Norat T, et al. Tobacco smoking and the risk of heart failure: A systematic review and meta-analysis of prospective studies. Eur J Prev Cardiol 2019;26:279-88.

33. Sepehripour AH, Lo TT, McCormack DJ, et al. Is there benefit in smoking cessation prior to cardiac surgery? Interact Cardiovasc Thorac Surg 2012;15:726-32.

34. Huded CP, Tuzcu EM, Krishnaswamy A, et al. Association Between Transcatheter Aortic Valve Replacement and Early Postprocedural Stroke. JAMA 2019;321:2306-15.

35. Reardon MJ, Adams DH, Kleiman NS, et al. 2-Year Outcomes in Patients Undergoing Surgical or SelfExpanding Transcatheter Aortic Valve Replacement. J Am Coll Cardiol 2015;66:113-21.

36. Smith CR, Leon MB, Mack MJ, et al. Transcatheter versus surgical aortic-valve replacement in high-risk patients. $\mathrm{N}$ Engl J Med 2011;364:2187-98.

37. Yang TH, Webb JG, Blanke P, et al. Incidence and severity of paravalvular aortic regurgitation with multidetector computed tomography nominal area oversizing or undersizing after transcatheter heart valve replacement with the Sapien 3: a comparison with the Sapien XT. JACC Cardiovasc Interv 2015;8:462-71.

Cite this article as: Liang $\mathrm{Y}$, Wang W, Wang X, Hei F, Guan Y. A single-center analysis of outcomes, risk factors, and new valves in Asian patients treated with early transcatheter aortic valve implantation. Cardiovasc Diagn Ther 2021;11(4):967-979. doi: 10.21037/cdt-20-928 


\section{Supplementary}

Table S1 Impact of different valves on outcomes

\begin{tabular}{|c|c|c|c|c|}
\hline & P-value & $\mathrm{HR}$ & 95\% lower confidence limit & 95\%upper confidence limit \\
\hline \multicolumn{5}{|l|}{ Venus A } \\
\hline All-cause mortality & 0.980 & 1.012 & 0.416 & 2.462 \\
\hline Stroke & 0.973 & 0.980 & 0.295 & 3.255 \\
\hline Heart failure & 0.779 & 0.830 & 0.227 & 3.039 \\
\hline Readmission & 0.281 & 0.586 & 0.221 & 1.549 \\
\hline \multicolumn{5}{|l|}{ TaurusOne } \\
\hline All-cause mortality & 0.909 & 0.884 & 0.108 & 7.238 \\
\hline Stroke & 0.987 & 0 & 0 & - \\
\hline Heart failure & 0.987 & 0 & 0 & - \\
\hline Readmission & 0.223 & 2.217 & 0.615 & 7.988 \\
\hline \multicolumn{5}{|l|}{ VitaFlow } \\
\hline All-cause mortality & 0.314 & 0.353 & 0.047 & 2.683 \\
\hline Stroke & 0.624 & 1.487 & 0.304 & 7.270 \\
\hline Heart failure & 0.987 & 0 & 0 & - \\
\hline Readmission & 0.706 & 0.749 & 0.166 & 3.369 \\
\hline \multicolumn{5}{|l|}{ J-Valve } \\
\hline All-cause mortality & 0.193 & 1.871 & 0.729 & 4.801 \\
\hline Stroke & 0.895 & 1.112 & 0.231 & 5.339 \\
\hline Heart failure & 0.325 & 2.260 & 0.446 & 11.454 \\
\hline Readmission & 0.448 & 0.558 & 0.124 & 2.514 \\
\hline
\end{tabular}

*, significant values. HR, Hazard Ratio; NYHA, New York heart association. In verifying the impact of valves on outcomes, variables enrolled in Cox regression models. All-cause mortality: liver disease, bicuspid valve, NYHA $\leq$ II, age, postoperative heart failure, postoperative stroke. Stroke: age, diabetes, myocardial infarction, stroke history, hypertension. Heart failure: age, male, smoking history, ejection fraction, BMI, NYHA $\leq$ II, diabetes, hypertension. Readmission: age, myocardial infarction, bicuspid valve, syncope, NYHA $\leq$ II, diabetes, smoking history. 
Table S2 Paravalvular leakage of different TAVI valves

\begin{tabular}{|c|c|c|c|c|c|c|c|}
\hline Valves & Sapien XT $(n=9)$ & Venus-A ( $n=97)$ & TaurusOne $(n=17)$ & VitaFlow $(n=25)$ & J-Valve $(n=27)$ & Total number & $P$ value \\
\hline 1 week: number & 9 & 92 & 17 & 25 & 25 & 168 & \\
\hline n (\%) & $3(33.3)$ & $44(47.8)$ & $3(17.6)$ & $5(20.0)$ & 8 (32.0) & $63(37.5)$ & 0.029 \\
\hline 1 month: number & 8 & 69 & 12 & 20 & 19 & 128 & \\
\hline 1 year: number & 8 & 61 & 14 & 18 & 16 & 117 & \\
\hline n (\%) & $3(37.5)$ & $26(42.6)$ & $2(14.3)$ & $5(27.8)$ & $3(18.8)$ & 39 (33.3) & 0.174 \\
\hline \multicolumn{8}{|l|}{$>$ mild PVL } \\
\hline 1 week: number & 9 & 92 & 17 & 25 & 25 & 168 & \\
\hline n (\%) & $1(12.5)$ & $4(5.8)$ & $2(16.7)$ & 0 & 0 & $7(5.5)$ & 0.139 \\
\hline 1 year: number & 8 & 61 & 14 & 18 & 16 & 117 & \\
\hline n (\%) & 0 & $2(3.3)$ & 0 & 0 & $1(6.3)$ & $3(2.6)$ & 0.735 \\
\hline
\end{tabular}

TAVI, transcatheter aortic valve implantation; PVL, paravalvular leakage.

Table S3 Outcomes of different stages

\begin{tabular}{|c|c|c|c|c|c|}
\hline Stage & Time (year/month) & All-cause mortality, n (\%) & Stroke, n (\%) & HF, n (\%) & PPI, n (\%) \\
\hline Stage $1, n=45$ & 2012/9-2014/10 & 0 & $2(4.4)$ & $2(5.0)$ & $5(11.4)$ \\
\hline Stage $2, n=34$ & $2014 / 11-2015 / 11$ & $3(9.0)$ & $1(2.9)$ & $1(3.3)$ & $8(23.9)$ \\
\hline Stage $3, n=40$ & 2015/12-2016/12 & $4(10.1)$ & $3(7.5)$ & $2(5.0)$ & $5(12.5)$ \\
\hline$P$ value & & 0.178 & 0.773 & 0.862 & 0.321 \\
\hline \multicolumn{6}{|l|}{3 years } \\
\hline Stage $1, n=45$ & 2012/9-2014/10 & $6(14.0)$ & $4(10.1)$ & $3(7.5)$ & $6(14.4)$ \\
\hline$P$ value & & 0.873 & 0.232 & 0.804 & 0.313 \\
\hline
\end{tabular}

HF, heart failure; PPI, permanent pacemaker implantation. 
Table S4 Sensitivity analysis in TAVI

\begin{tabular}{|c|c|c|c|c|}
\hline & Total $(n=175)$ & Without aortic valve regurgitation $(n=170)$ & $\mathrm{HR}(95 \% \mathrm{Cl})$ & $P$ value \\
\hline All-cause mortality & $13(7.5)$ & $13(7.7)$ & $0.970(0.450-2.093)$ & 0.939 \\
\hline Stroke & $8(4.6)$ & $8(4.7)$ & $0.971(0.365-2.588)$ & 0.954 \\
\hline MI & $3(1.8)$ & $3(1.2)$ & $0.970(0.196-4.807)$ & 0.971 \\
\hline Valve thrombosis & $2(1.2)$ & $2(1.2)$ & $0.970(0.137-6.887)$ & 0.976 \\
\hline PPI & $24(13.9)$ & $24(14.3)$ & $0.971(0.551-1.709)$ & 0.918 \\
\hline Heart failure & $9(5.5)$ & $8(5.0)$ & $1.090(0.421-2.826)$ & 0.859 \\
\hline HAVB & $23(13.4)$ & $23(13.8)$ & $0.970(0.544-1.730)$ & 0.919 \\
\hline All-cause mortality & $23(16.3)$ & $23(16.9)$ & $0.963(0.540-1.717)$ & 0.899 \\
\hline Stroke & $12(9.8)$ & $12(10.2)$ & $0.961(0.432-2.140)$ & 0.923 \\
\hline $\mathrm{AF}$ & $18(10.8)$ & $17(10.5)$ & $1.027(0.530-1.994)$ & 0.936 \\
\hline Heart failure & $11(7.2)$ & $10(6.8)$ & $1.065(0.452-2.507)$ & 0.886 \\
\hline HAVB & $24(14.1)$ & $24(14.5)$ & $0.970(0.551-1.708)$ & 0.915 \\
\hline PPI & $27(17.0)$ & 27 (17.6) & $0.968(0.568-1.650)$ & 0.904 \\
\hline Readmission & $21(18.2)$ & $20(17.8)$ & $1.012(0.549-1.867)$ & 0.969 \\
\hline
\end{tabular}

Values are $\mathrm{n}(\%)$. AF, atrial fibrillation; $\mathrm{Cl}$, confidence interval; HAVB, high-grade atrioventricular block; HR, hazard ratio; MI, myocardial infarction; PPI, permanent pacemaker implantation; TAVI, transcatheter aortic valve implantation.
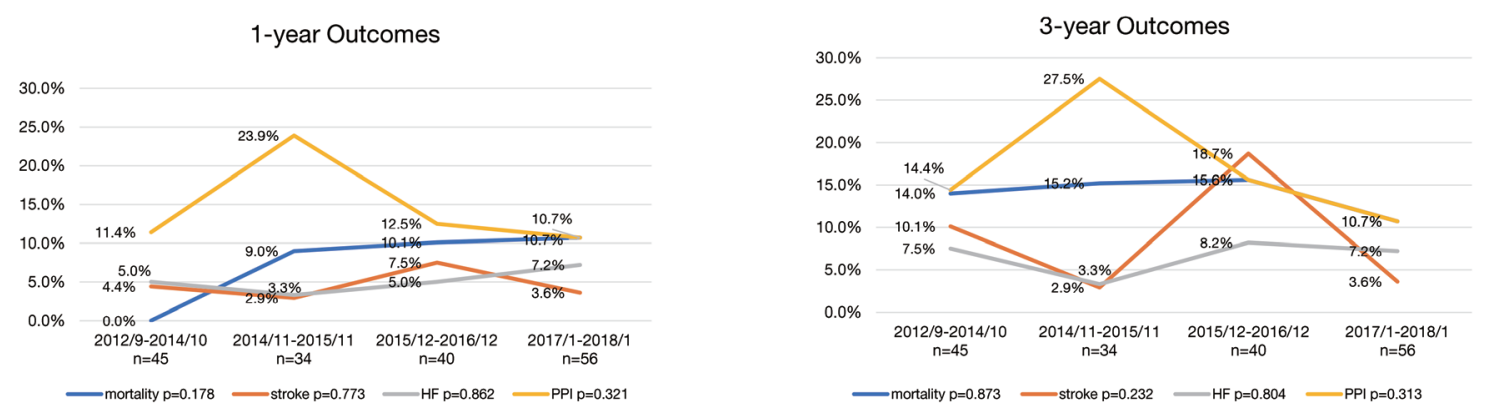

Figure S1 Outcomes of different stages. HF, heart failure; PPI, permanent pacemaker implantation. 